\title{
A Pipeline for Disaster Response and Relief Coordination
}

\author{
Pranav Maneriker \\ Nikhita Vedula \\ \{maneriker.1@,vedula.5\}@osu.edu \\ Ohio State University \\ Ethan Kubatko \\ Desheng Liu \\ \{kubatko.3,liu.738\}@osu.edu \\ Ohio State University
}

\author{
Hussein S. Al-Olimat \\ hussein@knoesis.org \\ Kno.e.sis Center, Wright State \\ University \\ Krishnaprasad Thirunarayan \\ Valerie Shalin \\ Amit Sheth \\ \{tkprasad,valerie,amit\}@knoesis.org \\ Kno.e.sis Center, Wright State \\ University
}

\author{
Jiayong Liang \\ Omar El-Khoury \\ \{liang.518,el-khoury.6\}@osu.edu \\ Ohio State University \\ Srinivasan Parthasarathy \\ srini@cse.ohio-state.edu \\ Ohio State University
}

\begin{abstract}
Natural disasters such as floods, forest fires, and hurricanes can cause catastrophic damage to human life and infrastructure. We focus on response to hurricanes caused by both river water flooding and storm surge. Using models for storm surge simulation and flood extent prediction, we generate forecasts about areas likely to be highly affected by the disaster. Further, we overlay the simulation results with information about traffic incidents to correlate traffic incidents with other data modality. We present these results in a modularized, interactive map-based visualization, which can help emergency responders to better plan and coordinate disaster response.
\end{abstract}

\section{CCS CONCEPTS}

-Applied computing; • Information systems $\rightarrow$ Spatial-temporal systems;

\section{KEYWORDS}

Storm Surge, Disaster Response, Spatio-temporal Modeling, Visualization, Traffic Data

\section{ACM Reference Format:}

Pranav Maneriker, Nikhita Vedula, Hussein S. Al-Olimat, Jiayong Liang, Omar El-Khoury, Ethan Kubatko, Desheng Liu, Krishnaprasad Thirunarayan, Valerie Shalin, Amit Sheth, and Srinivasan Parthasarathy. 2019. A Pipeline for Disaster Response and Relief Coordination. In Proceedings of the 42nd International ACM SIGIR Conference on Research and Development in Information Retrieval (SIGIR '19), July 21-25, 2019, Paris, France. ACM, New York, NY, USA, 4 pages. https://doi.org/10.1145/3331184.3331405

\footnotetext{
Permission to make digital or hard copies of all or part of this work for personal or classroom use is granted without fee provided that copies are not made or distributed for profit or commercial advantage and that copies bear this notice and the full citation on the first page. Copyrights for components of this work owned by others than the author(s) must be honored. Abstracting with credit is permitted. To copy otherwise, or republish, to post on servers or to redistribute to lists, requires prior specific permission and/or a fee. Request permissions from permissions@acm.org.

SIGIR '19, July 21-25, 2019, Paris, France

(C) 2019 Copyright held by the owner/author(s). Publication rights licensed to ACM. ACM ISBN 978-1-4503-6172-9/19/07 ...\$15.00

https://doi.org/10.1145/3331184.3331405
}

\section{INTRODUCTION}

Identifying standards and metrics for disaster resilience is a major challenge of considerable interest to federal agencies [13]. Communities identify expected changes in vulnerabilities to natural disasters and adapt their strategies accordingly [15]. Mitigating responses can be either pre- or post-disaster. Post-disaster response involves identifying the affected areas using multimodal data (e.g. unstructured text, location gazetteers, images), identifying the needs of the affected victims, and matching them with first responders [8].

In this work, we focus on the impact of floods and hurricanes induced by storm surge. Post-disaster response to these disasters requires image sources such as Synthetic Aperture Radar (SAR) imagery. We preprocess the images such that water corresponds to areas darker in intensity, and can be detected by an appropriate outlier detection framework (e.g. [10]). For forecasting, storm surge simulation results can be produced using the ADCIRC (ADvanced CIRCulation) model [4]. It is a finite element model used by a number of agencies and has been used for hindcasting prior hurricane events, as well as a predictive tool for designing/planning against future storms. Model predictions have shown good agreement with measured field data for a number of applications, including hindcasts of high water marks for many recent and historic storms [3]. By overlaying the predicted storm data on the true processed SAR image, we establish the correctness of the predictive storm models.

Additionally, the impact of the storm can be further quantified by retrieving co-located traffic incidents. To establish the importance of responding to storm predictions, we overlay, over the SAR image, traffic data that has been collected from the same time and geolocation range as the storm. The traffic data is a subset of a larger dataset collected by Moosavi et al. [11]. The dataset contains incidents related to flow congestion and accidents, which can be used as indicators of disaster impact.

\section{RELATED WORK}

Flood Mapping: Classical flood-mapping techniques rely upon various kinds of thresholding [14]. Recently, more advanced methods model the image as graphs and find flooded regions by using cut based, machine learning based and human input guided flood mapping [9]. In our work on flood mapping, we use a state-of-the-art 
approach which models the flood mapping problem in an attributed graph outlier detection framework [10].

Data Visualization: Previous work on visualizing structured input on maps has been done using multiple layers with different information $[7,8]$. Following a similar approach, our tool visualizes the data on water height, water velocity and traffic information into separate layers, which can be viewed independently and overlaid on each other. Users can also temporally navigate through multiple hours of data to visualize the evolution and progress of the storm.

Disaster forecasting: Forecasting for hurricane like disasters can be done with two major classes of models - statistical models that rely on global wind and forecasting patterns, and purely dynamical models that rely on the physics of fluid motions. The latter class is more popular today since they use finer spatial resolution and sophisticated physical process representation to improve the accuracy [16]. We utilize a finite element ADCIRC model, known to have good agreement with measured field data in a number of storms, such as Hurricanes Rita and Katrina [2, 6].

\section{METHOD}

Figure 1 shows the overall architecture and pipeline of our current disaster relief framework. This version does not show the social media data, but it is present in our previous work [8].
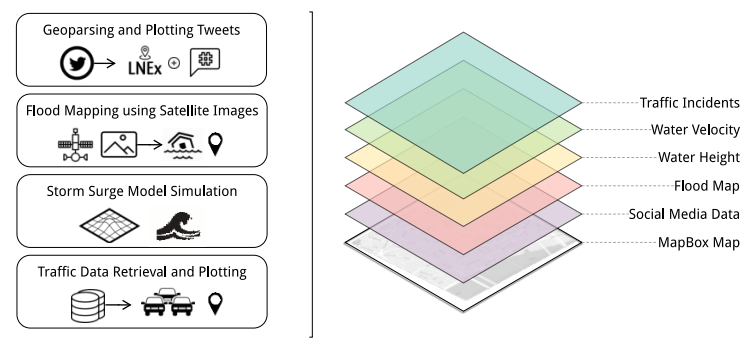

Figure 1: Left: Data generation. Right: Visualization layers.

\subsection{Data sources}

Synthetic Aperture Radar (SAR) imagery is suitable for flood mapping (predicting if an area is flooded or not) thanks to its ability to provide all-sky observations regardless of unfavorable illumination and weather conditions, and its sensitivity to water. The satellite images in this demo are acquired by the Sentinel-1 C-band SAR in the Interferometric Wide swath (IW) modes. The Sentinel-1 includes two identical satellites enabling a revisit time of 6 days. The spatial resolution reports adjacent targets $\Pi$ with a distance of 20 meters in range and 23 meters in azimuth direction, that are discernable. The image has two polarizations defined by the SAR signal direction, namely VV (vertically transmitted and vertically received) and VH (vertically transmitted and horizontally received). Collected Highresolution Ground Range Detected (GRDH) product is processed by using the Sentinel Application Platform (SNAP) from the ESA ${ }^{1}$, with standard procedures of radiometric calibration and terrain correction. The digital pixel values in GRDH are then converted

\footnotetext{
${ }^{1}$ European Space Agency
}

into calibrated backscatter intensity in decibel units. Elevation data from the National Elevation Dataset with a 1/3 arc-second spacing is used to correct SAR geometric distortion. After preprocessing, the SAR intensity is used for floodwater delineation. Given the smooth surface and high dielectric constant, water shows weaker SAR back scattering signal, thus corresponding to the darker areas in the intensity image.

The storm surge model domain used for simulations includes the Western North Atlantic, the Gulf of Mexico, and a large swath of the Texas floodplain (see Figure 2). The domain consists of approximately 6.5 million elements, with sizes ranging from approximately $17 \mathrm{~km}$ in the deep ocean down to $10 \mathrm{~m}$ along the coast. Meteorological forcing for the model, which includes wind and pressure fields, are obtained from Ocean Weather Inc. (courtesy of the Computational Hydraulics Group at UT Austin) and/or the National Hurricane Center's "best track" data [12]. Model output includes piecewise linear surfaces of the water surface elevation and depthaveraged velocities defined over the entire extent of the domain at time snaps of 1 hour.

Traffic events shown in this demonstration have been retrieved from a dataset which includes traffic events since Aug 2016 [11]. The dataset includes weather events, traffic accidents and congestions, and construction. For the purpose of the demonstration, we display two types of events on the geographical map likely to have been caused by a weather condition - accidents and congestion.
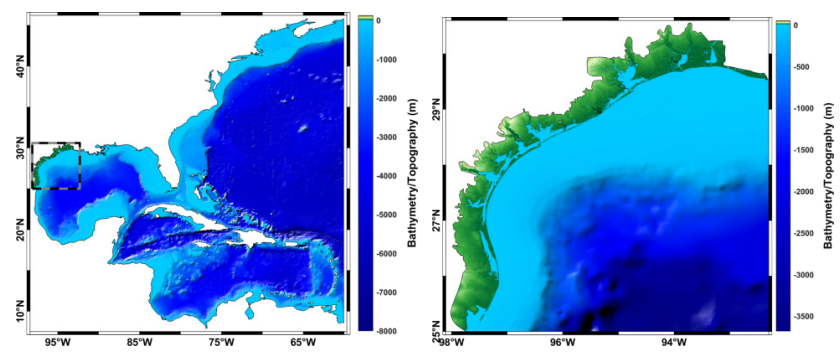

Figure 2: Left: Extent of model domain used. Right: Model details along the Texas coast.

\subsection{Web Visualization}

The storm surge model outputs triangulated meshes of vertices from which bathymetry (water depth) and velocity can be interpolated. However, each of these meshes is of very large size (file size $>100 \mathrm{MB}$ for each hour of data) and it is not feasible to load a new mesh into a web browser on every request. To overcome the size limitation, we use a simple sampling strategy to get an approximation of the mesh. We sample a regular grid of points within the bounds of the mesh. At each of the points, we compute the values of interest - bathymetry, velocity_x and velocity_y. Finally, we split these values into 256 levels, so that they can be encoded as (R, G, B) colours into an image. The image file generated from this process is used as a texture map for the visualization. The range of each of the parameters along with the bounds of the mesh is stored in a separate json file which can be loaded as needed. Using the texture map, the geometry of the visualization can be generated 

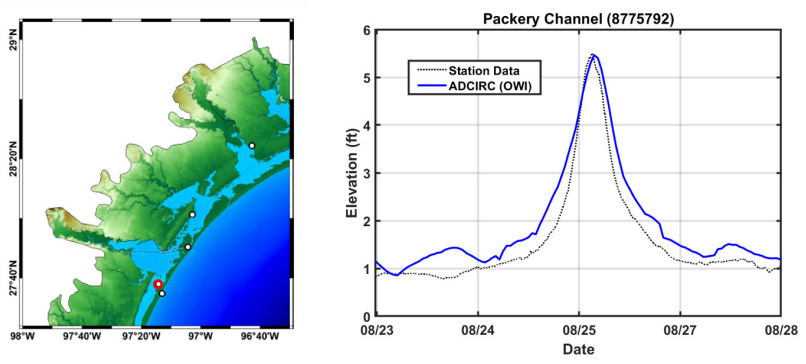

Figure 3: Right: Example results of the ADCIRC storm surge model output compared to recorded data at NOAA's Packery Channel station. Left: Station location shown in red.
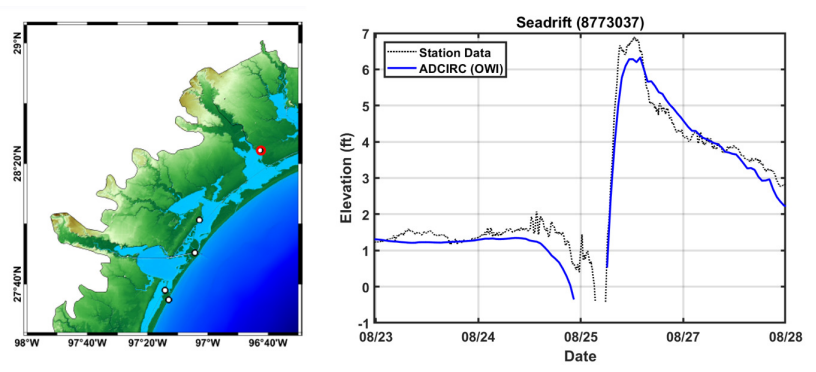

Figure 4: Right: Example results of the ADCIRC storm surge model output compared to recorded data at NOAA's Seadrift Channel station. Left: Station location shown in red.

with WebGL at run time with an image file $<1 \mathrm{MB}$ in size. This speeds up the visualization process.

The visualization is done using WebGL/ThreeJS ${ }^{2}$ based fragment shaders for the bathymetry, and 3D cones for the water velocity. In ThreeJS, it is possible to use BufferGeometry objects to map texture images to screen coordinates. We create a custom object composed of triangles, where each triangle is projected to the correct screen coordinates as per the Web Mercator projection [1]. We generate UV texture maps to correspond to the vertices so that the height texture is mapped to the correct regions on the Mapbox ${ }^{3}$ map.

\section{DEMONSTRATION}

In the demo presented here, we hope to aid the process of disaster response and relief. Users or first responders using our tool have access to structured traffic incident data, and can obtain information about the best evacuation routes. In general, our pluggable, modularized framework enables any spatio-temporal tagged data (e.g., tweets) extracted by an IR system to be added to its overlay visualization.

Figures 3 and 4 demonstrate the accuracy of the storm surge model for Hurricane Harvey. In this hindcasting, we see that the ADCIRC models capture the true water elevation accurately.

Figure 5 shows screenshots of all the controls offered in our framework. The different layers listed on the right can be toggled for viewing different aspects of the visualizations and a slider on

\footnotetext{
${ }^{2}$ https://threejs.org/

${ }^{3}$ https://www.mapbox.com/
}
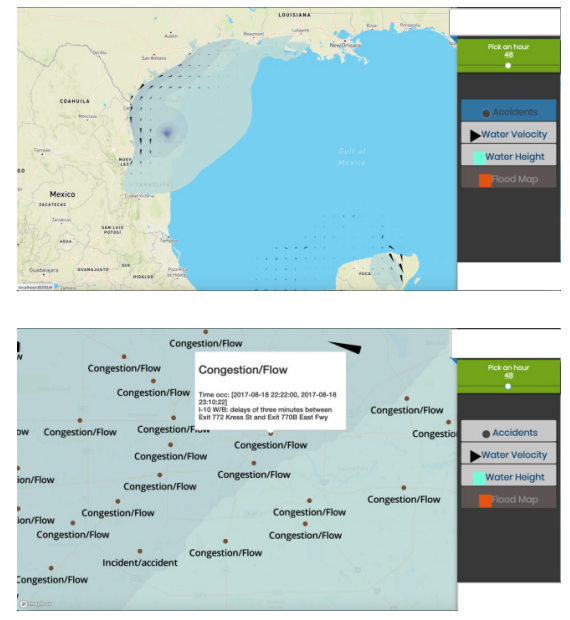

Figure 5: Top: Components of the interactive visualization. Bottom: Road accident information.
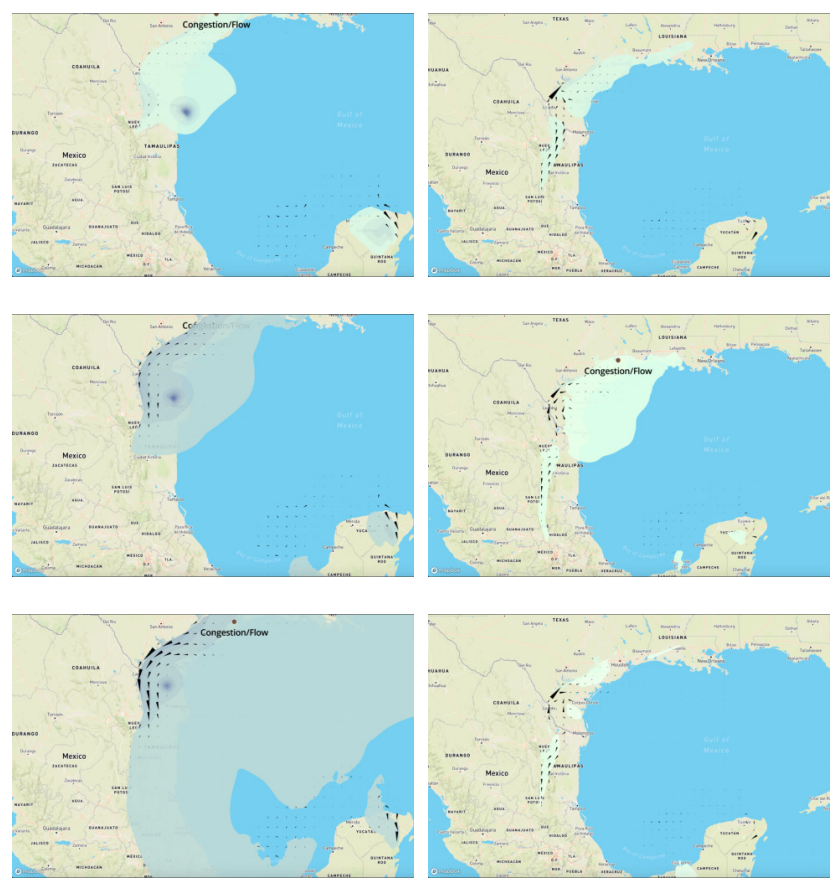

Figure 6: Storm visualization. left: Approach, Right: Retreat

the top-right can be used to scroll through different hours from the start of the storm. Darker (blue) colours (and longer length for wind cones) indicate a higher value. Finally, hovering over the markers provides information about traffic incidents at that location.

We overlay the visualization of the sampled storm surge data onto an interactive map. Figure 6 is a rendering of the evolution of the storm across the time frame.We can see some hotspots of high velocity and high depth in motion as the storm approaches land. 

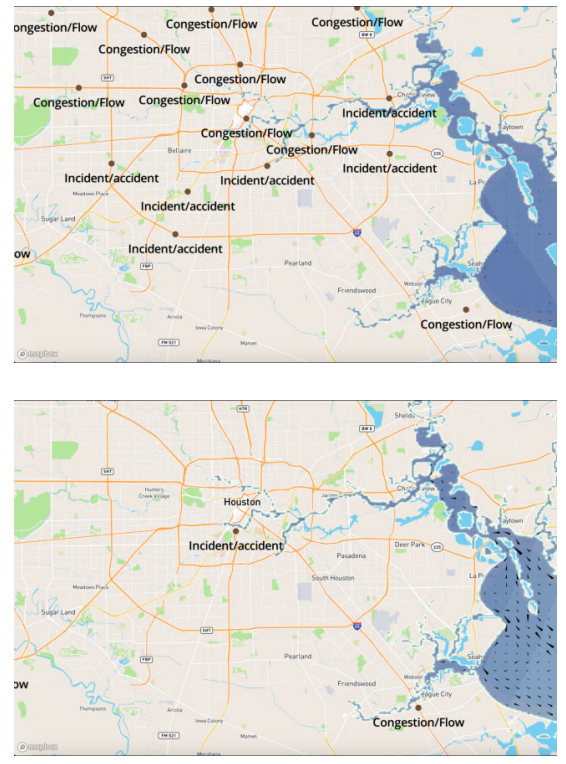

Figure 7: Top: Increased incidents during storm approach. Bottom: Fewer incidents during retreat.

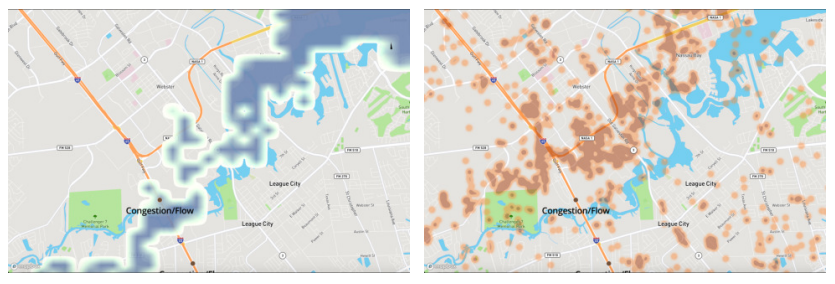

Figure 8: Left: Storm Surge. Right: Flood Map. Darker colours indicate higher water levels/higher flood impact

Next, we magnify the predictions of the storm surge model around the Houston area. We observe that there is an increase in traffic incidents initially as the storm moves towards the coast. They reach a peak around the midway point of the progress of the storm. The probable reason for this is that residents of this region begin evacuating, and finish evacuation over time. However, evacuation warnings were not issued for Harvey [5]. This may help explain the trends observed in the data. Figure 7 shows traffic incidents during the peak of the storm in comparison to the period that the storm was retreating.

Finally, we compare the post processed flood mapped data with the predictions on areas with high water elevation from the storm surge model. Figure 8 shows an example at high magnification levels in a coastal region. There is reasonable correspondence between the intermediate storm surge and final flood map data near the coast. But as we go farther away from the coast (bottom right of the map), the storm surge model has lowered resolution and cannot predict the flooded areas accurately.

\section{CONCLUSION}

Our proposed interactive visualization framework for disaster relief and response demonstrates the effectiveness of storm surge modelling as an effective tool for early warning systems. By overlaying traffic data, we are able to see the impact that the storm has as it evolves. Integrating this system with post-disaster flood maps as well as real-time information from social media can give rise to an effective disaster management and response framework.

\section{ACKNOWLEDGMENTS}

This work is supported by the National Science Foundation grant EAR-1520870. All content presented represents the opinion of the authors, and is not necessarily endorsed by their sponsors.

\section{REFERENCES}

[1] S E Battersby, M P Finn, E L Usery, and K H Yamamoto. 2014. Implications of web Mercator and its use in online mapping. Cartographica: The International fournal for Geographic Information and Geovisualization (2014).

[2] C Dawson, E J Kubatko, J J Westerink, C Trahan, C Mirabito, C Michoski, and N Panda. 2011. Discontinuous Galerkin methods for modeling hurricane storm surge. Advances in Water Resources (2011).

[3] J C Dietrich, C N Dawson, J M Proft, M T Howard, G Wells, J G Fleming, R A LuettichJr, J J Westerink, Z Lander, B O Blanton, C M Szpilka, and J H Atkinson. 2013. Real-time forecasting and visualization of hurricane waves and storm surge using SWAN+ADCIRC and FigureGen. Computational Challenges in the Geosciences 156 (2013), 49-70.

[4] J C Dietrich, S Tanaka, Joannes J Westerink, C N Dawson, R A Luettich, M Zijlema, L H Holthuijsen, J M Smith, L G Westerink, and H J Westerink. 2012. Performance of the unstructured-mesh, SWAN+ ADCIRC model in computing hurricane waves and surge. Fournal of Scientific Computing (2012).

[5] C Domonoske. [n. d.]. Why Didn't Officials Order The Evacuation Of Houston? NPR ([n. d.]). https://www.npr.org/sections/thetwo-way/2017/08/28/546721363/ why-didn-t-officials-order-the-evacuation-of-houston

[6] M E Hope, J J Westerink, A B Kennedy, P C Kerr, J C Dietrich, C Dawson, C J Bender, J M Smith, R E Jensen, M Zijlema, L H Holthuijsen, R A Luettich Jr, M D Powell, J Cardone, A T Cox, H Pourtaheri, H J Roberts, J H Atkinson, S Tanaka, H J Westerink, and L G Westerink. 2013. Hindcast and validation of Hurricane Ike (2008) waves, forerunner, and storm surge. Journal of Geophysical Research: Oceans (2013).

[7] P Simões Júnior, R Novais, V Vieira, L G Pedraza, M Mendonça, and K Villela. 2015. Visualization mechanisms for crowdsourcing information in emergency coordination. In Proceedings of the Brazilian Symposium on Human Factors in Computing Systems.

[8] S Kar, H S Al-Olimat, Thirunarayan, V L Shalin, A Sheth, and S Parthasarathy. 2018. D-record: Disaster Response and Relief Coordination Pipeline. In Proceedings of the 1st ACM SIGSPATIAL Workshop on Advances on Resilient and Intelligent Cities.

[9] J Liang, P Jacobs, and S Parthasarathy. 2018. Human-guided flood mapping: From experts to the crowd. In Companion of The 2018 Web Conference.

[10] J Liang, P Jacobs, J Sun, and S Parthasarathy. 2018. Semi-supervised embedding in attributed networks with outliers. In Proceedings of the 2018 SIAM International Conference on Data Mining.

[11] S Moosavi, M H Samavatian, A Nandi, S Parthasarathy, and R Ramnath. 2019. Short and Long-term Pattern Discovery Over Large-Scale Geo-Spatiotemporal Data. arXiv preprint arXiv:1902.06792 (2019).

[12] The National Hurricane Center (NHC). [n. d.]. NHC Data Archive. https://www. nhc.noaa.gov/data/. ([n. d.]). Accessed: 02-12-2019.

[13] SDR (Subcommittee on Disaster Reduction). 2005. Grand challenges for disaster reduction. (2005).

[14] N Otsu. 1979. A threshold selection method from gray-level histograms. IEEE transactions on systems, man, and cybernetics (1979).

[15] B Smit and J Wandel. 2006. Adaptation, adaptive capacity and vulnerability. Global environmental change (2006).

[16] H E Willoughby, E N Rappaport, and F D Marks. 2007. Hurricane forecasting: The state of the art. Natural Hazards Review (2007). 